\title{
Sur le vide d'une rencontre : Althusser lecteur de Machiavel
}

Sul vuoto di un incontro: Althusser lettore di Machiavelli

\section{Filippo Del Lucchese}

Traducteur : Fabio Bruschi

\section{Q OpenEdition}

\section{Journals}

Édition électronique

URL : http://journals.openedition.org/grm/623

DOI : $10.4000 / \mathrm{grm} .623$

ISSN : $1775-3902$

Éditeur

Groupe de Recherches Matérialistes

Référence électronique

Filippo Del Lucchese, «Sur le vide d'une rencontre : Althusser lecteur de Machiavel », Cahiers du GRM

[En ligne], 7 | 2015, mis en ligne le 05 juin 2015, consulté le 10 décembre 2020. URL : http://

journals.openedition.org/grm/623; DOI : https://doi.org/10.4000/grm.623

Ce document a été généré automatiquement le 10 décembre 2020.

(c) GRM - Association 


\title{
Sur le vide d'une rencontre : Althusser lecteur de Machiavel
}

\author{
Sul vuoto di un incontro: Althusser lettore di Machiavelli
}

Filippo Del Lucchese

Traduction : Fabio Bruschi

\section{NOTE DE L'ÉDITEUR}

Cet article, traduit de l'italien par Fabio Bruschi, a paru dans : M. Turchetto (éd.), Rileggere il capitale. La lezione di Louis Althusser, Venezia 9-10-11 novembre 2006, Atti del convegno parte seconda, Milan, Mimesis, 2009.

\section{Introduction}

1 La « rencontre » - ce mot « sans cesse » répété par Marx - pousse Althusser à se mesurer à Machiavel. Une rencontre pleinement "italienne", grâce à une beauté sicilienne éblouissante, en traversant la Romagne de César Borgia ${ }^{1}$ : d'abord Gramsci, mais tout de suite après Machiavel $^{2}$. Une rencontre aléatoire et contingente, et pourtant nécessaire. Surtout une rencontre bien étrange. Non pas en raison du «quoi », mais plutôt du " comment ", de la manière dont Althusser décide d'« épouser » la pensée de Machiavel. Althusser est un philosophe qui prend ses distances par rapport à la philosophie :

Plus je vais, plus je suis convaincu, à mon grand regret d'ailleurs que je ne suis pas un philosophe. Et pourtant il faudrait l'être, car il est possible et nécessaire, désormais, de l'être. Mais ce n'est pas pour moi. Je sais trop peu de choses, et n'ai plus le temps de les apprendre. Je suis un agitateur politique en philosophie. Il faut sans doute aussi ce genre de type, pour ouvrir les voies. D'autres, plus jeunes, mieux armés de connaissances en tout genre, seront le philosophe que je ne puis être ${ }^{3}$.

Donc «cet » Althusser tâche de faire de Machiavel - c'est-à-dire de quelqu'un qui n'est assurément pas un philosophe au sens traditionnel - précisément un philosophe, ou du 
moins un théoricien. Machiavel est étranger au monde des concepts "classiques » des philosophes ; ainsi s'ouvre le cours de 1962, le premier écrit qui lui est dédié4. À sa pensée - voici le paradoxe - qui est «riche» de sens théorique, les philosophes retirent toute portée théorique. C'est le défi à relever. Ceci pose la nécessité - dit Althusser - de redéfinir rien de moins que la " nature même » de l'objet théorique chez les classiques:

Ce que je voudrais suggérer c'est que la contradiction de cette reconnaissance pratique ou latente du sens théorique de la pensée de Machiavel d'une part, et le déni de toute portée théorique à la même pensée par les théoriciens eux-mêmes, cette contradiction est peut-être l'occasion et le moyen de poser le problème de la nature même de l'objet théorique chez les classiques. Si Machiavel a une valeur théorique, c'est la valeur théorique de l'objet de la théorie politique qui se trouve concernée, contestée dans ses prétentions, et en quelque sorte jugée par cette mise en question préalable qu'est cette théorie non reconnue de Machiavel $^{5}$.

Donc une rencontre paradoxale qui, dès les premières lignes, met en place une promesse engageante: une véritable redéfinition de l'«objet théorique». Cette promesse, importante et engageante, sera maintenue. Machiavel est une des vertèbres de l'épine dorsale de cette véritable redéfinition de la théorie et de la philosophie que sera le matérialisme aléatoire. Mais, d'une manière justement paradoxale, dans les écrits qu'Althusser consacre à Machiavel on trouve plutôt le «vide » d'une rencontre qui doit encore émerger. Il s'agit d'une lecture qui, en plus d'être fragmentaire et, dans beaucoup de cas, nullement originale, est souvent ambivalente. Pas originale et puissante comme elle le sera, au contraire, dans le dispositif global du matérialisme aléatoire.

\section{Impuissante solitude}

L'un des thèmes qui intéressent le plus Althusser, dans le cours de 62, est le rapport entre anthropologie et politique chez Machiavel, dans le contexte plus ample de la philosophie politique moderne. D'un côté, en ne parlant pas de l'homme ou de la nature humaine, mais des «hommes» au pluriel, Machiavel - c'est la conclusion peu pénétrante d'Althusser - ne construit pas une anthropologie politique comme celles que produiront Hobbes ou Spinoza. De l'autre côté, si l'on peut trouver chez Machiavel une forme d'anthropologie, elle n'a aucun lien explicite et direct avec la politique. L'usage «minimal » qu'en fait Machiavel correspondrait au refus d'un fondement éthique ou religieux des comportements sociaux. Mais l'anthropologie demeure "négative et critique », n'est jamais positive, c'est-à-dire qu'il n'y a aucune « déduction génétique des formes sociales et politiques à partir d'une théorie de la nature humaine $»^{6}$. Il s'agit de la "solitude de Machiavel », expression heureuse qu'Althusser utilisera ultérieurement et qui apparaît, donc, très précocement ${ }^{7}$. Il ne s'agit toutefois pas de la solitude d'une critique originale, mais d'un « échec ", d'une impuissance et d'une incapacité :

On ne saurait pourtant pas dire que cette solitude de Machiavel soit la solitude d'une critique. Machiavel n'est pas au-delà de l'opération théorique classique et de sa fondation, il est en deçà. Et l'on peut même considérer que l'échec de ses tentatives anthropologiques et historico-philosophiques témoigne plutôt d'une impuissance de fait, d'une incapacité à exprimer ce qu'il avait à dire dans des concepts philosophiques consacrés, que d'une véritable conscience critique ${ }^{8}$

Or, il serait possible de contester cette conclusion, dans une large mesure, sur la base à la fois du texte machiavélien et de son rapport avec les modernes, même en reconnaissant les différences évidentes de perspective chronologique. Cela signifie qu'il serait possible 
de montrer que Machiavel développe une anthropologie politique avec laquelle la modernité ressentira une profonde exigence de dialogue ${ }^{9}$.

Toutefois, au lieu d'opposer des textes machiavéliens à la lecture d'Althusser, il me semble plus utile de mettre en tension ses conclusions avec ses considérations plus générales sur la position de Machiavel dans l'histoire de la philosophie. La thèse de l'« échec » et de son «incapacité " peut en effet être lue à la lumière de la perspective différente dans laquelle le Secrétaire florentin est inséré dans certains textes plus tardifs. Prenons par exemple le célèbre et fulgurant parallèle qu'Althusser propose entre le Marx du Capital et le Machiavel du Prince dans «La solitude de Machiavel». En décrivant l'accumulation primitive, Marx a détruit l'illusion d'une histoire édifiante de l'origine et de la rencontre entre capital et travail ${ }^{10}$. De la même manière, Machiavel aurait décrit l'accumulation "primitive politique ", en réponse (théorique et non pas chronologique) aux théoriciens du droit naturel et de l'État moderne :

Je dirais que toutes proportions gardées Machiavel répond un peu de cette manière au discours édifiant que tiennent les philosophes du droit naturel sur l'histoire de l'État. J'irais jusqu'à suggérer que Machiavel est peut-être un des rares témoins de ce que j'appellerai l'accumulation primitive politique, un des rares théoriciens des commencements de l'État national. Au lieu de dire que l'État est né du droit et de la nature, il nous dit comment doit naître un État s'il veut durer, et être assez fort pour devenir l'État d'une nation ${ }^{11}$.

Althusser semble ne pas se rendre compte - au sein de cette comparaison puissante - que la démystification et le refus par Marx de l'individu bourgeois, du travailleur indépendant et de son esprit économe, correspond largement à la démystification et au refus par Machiavel d'une " anthropologie » au sens classique du terme. Lorsqu'Althusser parle en 62 de l'« échec » de Machiavel, il ne semble pas considérer que les bases de cet échec sont précisément ce qui permet de rapprocher, du point de vue théorique, son réalisme de celui de Marx, comme il le fera justement dans «La solitude de Machiavel ». Et ceci est d'autant plus curieux si l'on pense que la réponse la plus puissante à cet individu abstrait, dans la modernité, sera bien celle de Spinoza, fondée explicitement sur le réalisme anthropologique de Machiavel ${ }^{12}$.

Prenons un autre exemple. Althusser se concentre sur la lecture que Machiavel fait de Polybe afin de sortir d'une philosophie de l'histoire circulaire et cyclique, dans laquelle le destin des États serait déjà inscrit dans leur origine. Un thème, donc, qui recèle une importance capitale pour tout marxiste. Althusser met justement l'accent sur la prise de distance que Machiavel accomplit par rapport à Polybe : les États ne parcourent pas de manière cyclique leur parcours de génération et de corruption des différentes formes de gouvernement parce qu'ils rencontrent sur leur chemin d'autres États plus forts et puissants - c'est-à-dire la guerre - qui interrompent ce cycle, qui serait en soi infini ${ }^{13}$. L'élément d'originalité le plus fort dans l'usage hétérodoxe que Machiavel fait de Polybe est la liaison entre guerre et «lutte des classes». La différence de puissance entre les États qui s'affrontent dépend directement du conflit interne entre les classes, entre le peuple et les grands ${ }^{14}$. Ce qui est vraiment nouveau dans l'analyse de Machiavel est le fait de lier de manière indissoluble (ou, pour mieux dire, de démasquer l'« idéologie » de ceux qui voudraient séparer) guerre externe et conflit interne des classes ${ }^{15}$.

Or, non seulement Althusser ne saisit pas l'originalité de cet argument, mais il affirme que la force de Machiavel consiste dans la valorisation du "gouvernement mixte». L'union des trois bonnes formes de gouvernement - en les neutralisant en tant que formes singulières - permet de sortir du cercle polybien et de sa philosophie de l'histoire : 
La théorie des cycles est remise en question par la constitution de ce gouvernement mixte qui réunit les avantages des trois bons gouvernements : prince + grands + peuple. Comment interpréter cette synthèse sinon comme une synthèse qui permet l'espoir d'échapper à la loi de l'infinité elle-même dans la constitution du gouvernement qui combine (en les neutralisant mutuellement) les principes spécifiques et bénéfiques des trois gouvernements fondamentaux? Je dirais donc: l'infinité du cycle de l'histoire est abstraite. Le concret c'est la lutte des États entre eux qui interrompt l'infinité du cycle, jusqu'à ce que survienne cet État mixte si bien composé qu'il est lui-même comme un défi à l'infinité du cycle ${ }^{16}$.

Sur le plan théorique, c'est bien connu, celle-ci est déjà la lecture de Polybe et, avant lui, d'Aristote $^{17}$. Dans la lecture d'Althusser manque donc toute référence à l'originalité de Machiavel par rapport à ses sources classiques, qui émerge pourtant avec force de sa théorie du conflit.

Qui plus est, à la différence de ce qui a été dit concernant l'anthropologie, dans les écrits postérieurs cette lecture ne semble pas changer significativement. La solution est encore une fois une "synthèse " des trois formes à travers laquelle Machiavel sortirait de l'argumentation de Polybe :

Telle est, dans sa simplicité, la théorie cyclique de l'histoire, la typologie des gouvernements, telle que Machiavel l'a, en partie, empruntée à Polybe. Par cette troisième thèse de la nature cyclique de l'histoire, Machiavel semble avoir réalisé, réussi une "synthèse ", au sens hégélien-vulgaire, entre la première thèse (le cours immuable), et la seconde thèse (la mobilité universelle). Qu'est-ce en effet que le cycle de l'histoire, sinon le mouvement immobile, l'immuable mouvement de la répétition des mêmes changements ? ${ }^{18}$

L'allusion - d'autant plus énigmatique si l'on mesure correctement la valeur des mots employés par Althusser - faite à un langage qui renvoie à des concepts aristotéliciens ne peut pas nous échapper. Ce qu'Althusser semble reprocher à Machiavel - le fait de ne pas avoir su ou pu exprimer entièrement sa pensée révolutionnaire - semble se refléter sur ses propres analyses: Althusser entrevoit l'originalité de Machiavel - et c'est ici que commence le rapport «intime » qui le mènera jusqu'à l'« identification » -, mais ne la trouve pas, paradoxalement, dans ses textes, en se bornant à une interprétation aucunement originale.

Le discours semble différent pour l'ensemble des écrits plus tardifs, qui ont été réélaborés jusqu'en 1986, recueillis dans le deuxième tome des Écrits philosophiques et politiques sous le titre «Machiavel et nous ». Mais il est différent seulement en apparence, car ici, d'un côté, ce Machiavel est beaucoup plus mûr, puissant et commence à être lu dans la perspective du dernier Althusser, celui du matérialisme aléatoire. D'un autre côté, toutefois, cette projection sur le matérialisme aléatoire est souvent seulement apparente et se maintient sur un niveau purement superficiel.

11 Althusser parle de la pensée de Machiavel comme d'une pensée de la "conjoncture singulière ", du " cas singulier aléatoire ", de la "détermination dialectique aléatoire ». Mais les notes d'édition de François Matheron rappellent opportunément qu'il s'agit avec une régularité inflexible - d'«ajouts manuscrits successifs ». Donc tout se passe comme si Althusser relisait ces notes stratifiées avec des lentilles différentes de celles portées précédemment, en «collant» le langage et les catégories du matérialisme aléatoire sur sa lecture précédente de Machiavel. Ceci est intéressant parce qu'en réalité ce qu'Althusser décrit dans ces textes est significativement différent et coïncide seulement de manière superficielle avec ce véritable « arsenal » théorique qui répond au 
nom de Machiavel, et avec le rôle et le statut que celui-ci aura dans le dispositif du matérialisme de la rencontre.

12 À travers le langage puissant et évocateur du matérialisme aléatoire, on tente ici de décrire un objet théorique "distant», comme dans cette quasi-définition qu'offre Althusser de la "pensée dans la conjoncture", c'est-à-dire de ce que signifie, pour Machiavel, penser dans la conjoncture le problème de l'unité d'un État national italien :

Que signifie penser dans la conjoncture? Penser un problème politique sous la catégorie de conjoncture? Cela signifie d'abord tenir compte de toutes les déterminations, de toutes les circonstances concrètes existantes, en faire le recensement, le décompte et la comparaison (...). Mais ce recensement des éléments et des circonstances ne suffit pas. Penser sous la catégorie de conjoncture, ce n'est pas penser sur la conjoncture, comme on réfléchirait sur un ensemble de données concrètes. Penser sous la conjoncture, c'est littéralement se soumettre au problème que produit et impose son cas [ajout manuscrit tardif] : le problème politique de l'unité nationale, la constitution de l'Italie en État national (...). Machiavel ne fait qu'enregistrer dans sa position théorique un problème qui est objectivement, historiquement posé par le cas de [ajout manuscrit tardif] la conjoncture : non par de simples comparaisons intellectuelles, mais par l'affrontement des forces de classes existantes, et leur rapport de développement inégal, en définitive par leur avenir aléatoire [ajout manuscrit tardif] ${ }^{19}$.

D’autres exemples tirés de "Machiavel et nous » méritent de retenir notre attention. Le premier, qui frappe tout particulièrement, est constitué par les différentes manières dont Althusser parle de Machiavel en utilisant les catégories aristotéliciennes de «matière et forme ». Machiavel lui-même emploie ce langage mais, à nouveau, seulement le langage et non pas les concepts qui, dans une stratégie rhétorique qui sera chère à Spinoza, sont au contraire renversés. Pour Machiavel (dans ce cas un véritable "fils de la terre" s'opposant aux " amis des formes ») semble se donner - contre Aristote - une véritable primauté de la matière sur la forme. Une matière saine ou une matière corrompue offrent "l'occasion " aux acteurs politiques de démontrer leur vertu ${ }^{20}$. La matière est alors tout autre chose que l'élément passif d'Aristote: Florence est une matière apte à être ordonnée en République et qui voudrait faire l'inverse, c'est-à-dire lui imposer une forme différente, serait nécessairement mené à sa propre ruine. Ce n'est pas par hasard que le couple matière/forme est utilisé prioritairement par Machiavel avec celui de fortune/ occasion $^{21}$.

Althusser s'exprime de la manière la plus claire, dans ce sens, dans le cours de 62 , en séparant nettement Machiavel des catégories ou concepts aristotéliciens :

La «matière " politique dont parle Machiavel quand il a en vue la situation italienne n'est même pas comparable à la puissance aristotélicienne, qui est à la fois manque de sa forme, mais aspiration à sa forme, et en contient (comme le bloc de marbre où certaines veines suggèrent la forme que lui donnera le sculpteur) l'esquisse future. Elle est encore moins comparable à la forme intérieure contenue par le moment hégélien de l'histoire (qui mûrit en lui, sans le savoir, la forme implicite qui, une fois rejetée l'ancienne forme existante, apparaîtra dans l'avènement de la nouvelle époque). Non : la matière est pur vide de forme, pure attente informe de forme. La matière italienne est une puissance vide, qui attend $d u$ dehors qu'une forme lui soit apportée et imposée ${ }^{22}$.

Il s'agit de la reconnaissance - ajoute Althusser - de la «contingence radicale » dans laquelle la forme nouvelle est "appliquée » à la matière existante. La nécessité d'une forme nouvelle a comme condition la contingence radicale d'un nouveau commencement. 

postérieurs par une attitude plus ambivalente et nuancée. Quelle est la forme - se demande Althusser - sous laquelle réunir toutes les forces positives à disposition pour réaliser l'objectif politique de l'unité nationale? Une telle forme est le prince, un individu exceptionnel, doté de vertus exceptionnelles, qui dans des circonstances exceptionnelles sera capable de mobiliser les forces nécessaires ${ }^{23}$. C'est presque une philosophie de l'histoire qui émerge de ce prince machiavélien :

Le prince de Machiavel est un souverain absolu à qui l'histoire " confie une tâche " décisive : celle de "donner forme " à une " matière " existante, matière aspirant à sa forme, la nation. Le prince nouveau de Machiavel est donc une forme politique définie chargée de réaliser les exigences historiques «à l'ordre du jour»: la constitution d'une nation ${ }^{24}$.

Derrière ces considérations il y a, bien entendu, la lecture de Gramsci et du "prince nouveau ». Tout en invitant à « peser » ces mots de Gramsci, Althusser partage et met en valeur son entreprise politique et culturelle : "Voilà, sous la nuit du fascisme, comment Machiavel "parle" à Gramsci : au futur. Et le prince moderne projette alors sa lumière sur le prince nouveau: Gramsci écrit calmement que Le Prince est un "manifeste", et une "utopie révolutionnaire". Disons, pour condenser: "un manifeste utopique révolutionnaire" $»^{25}$.

d'un côté, toute la charge philosophique de ce langage ne peut pas échapper à Althusser ; d'un autre côté, il semble au contraire prêt à rabattre Machiavel sur la lecture de Gramsci. Si Althusser s'éloigne de Gramsci par beaucoup d'aspects, par exemple sur l'idée de compénétration entre force et consensus ${ }^{26}$, ici il semble au contraire l'accueillir pleinement, en allant jusqu'à dire que «si les nouveaux rapports à la théorie et à l'Antiquité que nous venons d'analyser sont originaux et formellement féconds, il reste qu'ils ne sont pas dénués d'une certaine illusion : l'illusion utopique. S'il est vrai que toute utopie cherche dans le passé la garantie et la forme de l'avenir, Machiavel, qui cherche dans Rome la solution future au problème politique de l'Italie, n'échappe pas à l'illusion de l'utopie $»^{27}$. faiblesses de la lecture althussérienne de Machiavel. Mais je crois qu'il est plus intéressant, parvenus à ce point, de poser une question différente à ces textes: abandonner pour un instant la lecture, pour mettre au centre le problème théorique qui émerge de la rencontre entre Althusser et Machiavel. Il faut consulter, écrit Althusser, «non plus [la] simple littéralité formelle [des textes de Machiavel], mais leur fonctionnement $\|^{28}$. Il est peut-être opportun, de la même manière, de laisser la «littéralité formelle» des textes d'Althusser afin de considérer leur fonctionnement théorique. Pour ce faire, la meilleure manière est justement de passer par Gramsci. D'un côté, parce que, en montrant les limites de sa lecture, on en oublie souvent les potentialités. De l'autre côté, parce que ces mêmes potentialités ont une importance théorique aussi bien qu'historiographique. Et précisément du «creuset » de Machiavel, Gramsci et Althusser tirent des éléments théoriques d'une importance extraordinaire.

On insiste souvent sur la linéarité de la lecture gramscienne et sur les difficultés qu'elle comporte de par la «traduction » ou l'emploi politique du prince de Machiavel dans la perspective du Parti politique. Un exemple typique de cette traduction problématique pourrait être le lien établi par Gramsci entre les Jacobins et le contexte de la Physiocratie, en se demandant si Machiavel aurait en quelque sorte « devancé son temps » et anticipé

Cahiers du GRM, 7 | 2015 
quelques exigences qui ont ensuite trouvé leur expression justement chez les Physiocrates ${ }^{29}$. Toutefois - et en prenant en compte ici aussi le caractère stratifié et composite de ces textes -, l'excessive « linéarité » qui émerge du Machiavel de Gramsci et qui a sans doute attiré l'attention d'Althusser - n'est pas aussi banale ou naïve que l'on pourrait croire à première vue. Il suffit de penser que si l'intention d'Althusser est de faire de Machiavel un philosophe, c'est précisément sur ce point que s'était développée la critique gramscienne de la position de Croce et sa réévaluation de la "politiquepratique » de Machiavel et, avec lui, de Marx ${ }^{30}$.

Ensuite, du point de vue d'une conception de l'histoire, de son ouverture et des possibilités d'intervenir en elle - thème cher, pour des raisons différentes, à la fois à Machiavel et à Althusser - la lecture gramscienne de la vertu comme principe de l'intervention dans la « conjoncture » n'est aucunement naïve. À propos de l'organisation militaire, Gramsci écrit :

L'élément décisif de toute situation est la force organisée en permanence et préparée de longue main que l'on peut faire avancer quand on juge qu'une situation est favorable (et elle ne l'est que dans la mesure où une telle force existe et où elle est pleine d'ardeur combative); c'est pourquoi la tâche essentielle consiste à veiller systématiquement et patiemment à former une telle force, à la développer, la rendre toujours plus homogène, compacte, consciente d'elle-même. Cela se voit dans l'histoire militaire, et dans le soin avec lequel on a toujours fait en sorte que les armées soient prêtes à commencer une guerre, à n'importe quel moment. Si les grands États ont été de grands États, c'est précisément parce qu'ils étaient toujours prêts à intervenir efficacement dans les conjonctures internationales favorables, et si celles-ci étaient favorables, c'est parce qu'il y avait la possibilité concrète d'y intervenir efficacement ${ }^{31}$.

On peut difficilement imaginer une description plus proche de l'idée machiavélienne d'intervention de la vertu en conjoncture. Toute la réflexion machiavélienne sur l'entrecroisement entre politique et guerre, en particulier dans l'Art de la guerre, vise à développer cette idée d'une préparation des conditions les meilleures (jamais parfaites, mais seulement les meilleures) d'une intervention dans la conjoncture ${ }^{32}$. Et il est encore plus significatif que Gramsci parle de cette conception à travers une référence à Albert Mathiez qui, dans son interprétation de la Révolution française, nie justement la linéarité naïve, dans l'histoire, des rapports entre crises et ruptures ${ }^{33}$.

Nous sommes donc bien loin de la tentative d'utiliser «Machiavel » comme un simple instrument pour définir un parcours linéaire et esquisser une philosophie vulgaire de l'histoire ${ }^{34}$. Je me demande si, lorsqu'Althusser parle de conjoncture dans ses textes sur Machiavel - quelque chose de bien différent, à nouveau, de la « conjoncture » qui apparaît dans les écrits sur le matérialisme aléatoire -, il ne se rapproche et n'exprime pas quelque chose de très proche de ce dont parle ici Gramsci. À travers ces textes, il serait alors possible de voir, d'un côté, un Gramsci mieux différencié de la conception vulgaire et linéaire de la philosophie de l'histoire avec laquelle il est souvent confondu, et de l'autre, un Althusser encore en train de forger cet instrument théorique extrêmement précieux que sera le Machiavel du matérialisme aléatoire.

Mais l'ambivalence relative à la conception du prince et le nœud problématique qui en dérive est encore plus intéressante. Ici, avec la conception du parti comme prince moderne, nous n'en sommes même plus à une interprétation de Machiavel, mais à un usage « instrumental » qui semble à nouveau l'écraser totalement sous le problème politique contemporain de Gramsci. Pourtant, cet usage si actualisant et politiquement orienté semble produire, de manière paradoxale, des effets théoriques intéressants. Des 
effets qui émergent tout à la fois de la lecture d'Althusser, et du rôle qu'Althusser joue ou peut jouer dans le débat philosophico-politique contemporain.

\section{Le prince, principe partial ${ }^{35}$}

\section{représente, "plastiquement " et "anthropomorphiquement ", le symbole de la volonté collective :}

Le prince moderne, le mythe-prince, ne peut être une personne réelle, un individu concret, il ne peut être qu'un organisme, un élément complexe de société dans lequel a commencé déjà de se concrétiser une volonté collective qui s'est reconnue et affirmée en partie dans l'action. Cet organisme est déjà donné par le développement historique et c'est le parti politique, première cellule dans laquelle se concentrent des germes de volonté collective qui tendent à devenir universels et totaux ${ }^{36}$.

Les Jacobins - voici le parallélisme dressé par Gramsci - furent une «incarnation catégorique » du prince de Machiavel et la «théorie » du prince nouveau devrait contenir une partie consacrée précisément à la volonté politique et collective, à sa formation concrète, à son affirmation historique et partiale, au sens de la partie, d'une partie, et non pas du tout de la politique. Gramsci vise ici Sorel et un type de spontanéité révolutionnaire destinée à l'échec et à la dissolution. Outre l'attaque manifeste contre Sorel (et l'évitement significatif de la part de Gramsci de l'expression rousseauiste de "volonté générale», à laquelle il préfère celle de "volonté collective»), cette interprétation a un évident et profond intérêt théorique en plus d'un mérite historiographique, qui n'a rien à voir avec le problème politique de l'organisation et du parti et qui le dépasse largement. Son intérêt est de mettre en relief comment le prince, pour Machiavel, est un élément partial, qui ne représente pas le tout, mais qui est au contraire une partie parmi et contre d'autres parties, un acteur dans la lutte. Sa vertu se déploie non pas dans l'organisation d'un principe supérieur qui se hisse au-dessus du conflit et l'organise, de manière transcendante, mais seulement à l'intérieur et à travers le conflit, de manière radicalement immanente ${ }^{37}$.

Or, l'importance de cette conception va bien au-delà de l'interprétation de Machiavel. Dans le débat philosophico-politique récent, en effet, a été développée une discussion qui porte justement autour d'Althusser lecteur de Machiavel et, en particulier, de la conception et du rôle du prince. Même sur ce point la lecture d'Althusser semble particulièrement ambivalente. Le Prince comme manifeste politique, l'importance du "déséquilibre » énoncé dans le proemio, c'est-à-dire la distance sur laquelle s'appuie avec violence - Machiavel, l'écrivain qui appartient au peuple, pour parler des princes; la cohérence théorique entre Le Prince et les Discours: tous ces thèmes montrent qu'Althusser - ainsi que Gramsci - a bien saisi le lien et la valeur en même temps philosophique et politique de Machiavel. Le prince est de manière irréductible un principe partial. Et c'est un principe pluriel, multiple, collectif et conflictuel. Car il ne représente pas le peuple (en le réduisant à une unité comme le souverain hobbesien) : le prince est le peuple contre les grands ${ }^{38}$.

D'un autre côté, toutefois, Althusser semble glisser, parfois, vers ce qui a été appelé, peutêtre avec une certaine exagération, un "écho volontariste $"^{39}$. Dans Machiavel et nous, Althusser écrit : 
Machiavel se place certes du point de vue du peuple, mais ce Prince, auquel il assigne la mission d'unifier la nation italienne, s'il doit devenir un Prince populaire, n'est pas lui-même peuple. De même le peuple n'est pas appelé à devenir Prince. Il y a donc une dualité irréductible entre le lieu du point de vue politique et le lieu de la force et de la pratique politique, entre le «sujet " du point de vue politique, le peuple, et le «sujet» de la pratique politique, le Prince. Cette dualité, cette irréductibilité, affecte et le Prince et le peuple. Le Prince étant défini uniquement, exclusivement, par la fonction qu'il doit accomplir, c'est-à-dire par le vide historique qu'il doit remplir, est une forme vide, un pur possible-impossible aléatoire: aucune appartenance de classe ne le dispose à remplir sa tâche historique, aucun lien social ne le rattache à ce peuple qu'il doit unifier en nation ${ }^{40}$.

À nouveau, ici, « aléatoire » est un ajout successif. Mais ce qui pose surtout problème est justement le concept de "vide » rattaché à la figure du prince, volonté «absolue » qui émerge et s'affirme sur le «vide» des conditions historiques. Le prince est en quelque sorte « soustrait » à la dynamique conflictuelle, à la rencontre contingente des éléments en lutte pour en faire précisément un «pur» principe. En distinguant le manifeste machiavélien de celui de Marx et Engels, Althusser - de manière justement ambivalente soutient que si celui-ci est interne à un point de vue de classe, celui-là maintient une distance par rapport à la perspective de classe, puisqu'en même temps le prince ne doit pas devenir peuple et le peuple ne doit pas devenir prince. La pratique politique, dans ce sens, s'établit dans un espace vide qui doit rester vide ${ }^{41}$.

Or, il me semble que la meilleure manière d'interpréter ces textes est d'en conserver les ambivalences, la complexité et la stratification, sans assimiler Althusser à un Machiavel inexistant pour faire de ces deux penseurs un instrument théorique d'une "démocratie sans conflit ». Il en résulterait en effet l'oblitération du legs le plus puissant et original de Machiavel : précisément l'idée que la politique est toujours et seulement affirmation partielle et conflictuelle, que le prince opère seulement à l'intérieur et à travers la conjoncture, sans jamais pouvoir déterminer, de l'extérieur et de manière transcendante, cette conjoncture. Emmanuel Terray, par exemple, a écrit qu'Althusser a parfaitement compris ce que Machiavel entend par « fonder sur le vide » et la « solitude » du fondateur ${ }^{42}$. Fonder sur le vide signifie se détacher du conflit et dépasser la violence originaire naturelle pour fonder l'État ${ }^{43}$.

Bien plus articulé est le concept de "démocratie radicale » élaboré par Miguel Vatter et basé précisément sur le vide, sur la « solitude » et sur la distance du prince machiavélien à l'égard de la politique et du conflit ${ }^{44}$. Machiavel - écrit Vatter - soutient qu'il existe une différence substantielle entre le désir des grands de dominer et le désir du peuple de ne pas être dominé ${ }^{45}$. La politique populaire serait donc en deçà de toute forme politique, de toute volonté de domination, de tout projet constitutif. Elle serait un pur retrait dans une zone de neutralité, d'indifférence, de «liberté négative ». Autrement dit, la puissance du peuple serait l'envers de la puissance spinozienne ${ }^{46}$. Une puissance qui demeure indifférente à son propre accomplissement, un pur possible dont le désir est seulement celui de ne pas être gouverné.

Pour définir ce type de puissance, Vatter convoque d'un côté la traduction arendtienne d'isonomie par l'idée de "no-rule » et de l'autre l'idée de Giorgio Agamben, développée dans Homo sacer, selon laquelle la possibilité ou la puissance devrait être pensée en termes non aristotéliciens, c'est-à-dire de telle sorte qu'elle soit indifférente au passage à l'acte. Vatter fait émerger cette "in-différence souveraine du peuple au gouvernement » chez Machiavel et, en particulier, chez le Machiavel d'Althusser, en faisant signe vers une 
démocratie possible qui ne soit pas une forme de "gouvernement ", dans le sens du kratos , c'est-à-dire qui ne soit pas une forme de domination, mais un concept nouveau de liberté politique dans un contexte post-marxiste. "Chez Machiavel - écrit Vatter - le Prince nouveau n'est finalement rien d'autre que le prince civil, qui est appelé ainsi précisément parce qu'il fonde l'État en restant séparé de la radicalité du conflit social à travers l'institution d'une société civile (...). Le pouvoir constitué fonde ainsi soi-même et devient "l'accomplissement" du pouvoir constituant, en rendant possible la société civile. Une société devient "civile" en pacifiant le conflit social qui la constitue $»^{47}$.

31 Le passage du prince «civil » à la société civile - passage, qui plus est, basé sur la «pacification » du conflit qui l'a fait surgir - est une interprétation qui, dans le meilleur des cas, force excessivement le texte de Machiavel. L'originalité de celui-ci consiste au contraire justement dans le développement d'une théorie du conflit qui ne peut ni préfigurer le conflit naturel du jusnaturalisme qui lui succède ni, encore moins, trouver une issue dans la création d'une « société civile ». La lecture d'Althusser, comme on l'a vu, laisse ouvertes dans ses ambivalences des marges assez larges d'interprétation. Mais il est totalement incongru d'effacer ces ambivalences pour en faire émerger une lecture de Machiavel qui oblitère la théorie du conflit.

32 La puissance théorique de Machiavel émerge sans ambivalences, chez Althusser, dans les écrits sur le matérialisme aléatoire, bien plus donc que dans les écrits sur Machiavel luimême. La rencontre d'Althusser avec Machiavel - écrit encore Terray - a d'abord lieu à travers Montesquieu et la réaction du XVIII siècle au contractualisme et ensuite à travers Gramsci. Machiavel aurait pu également être rencontré à travers Spinoza, dit Terray, mais il n'y a aucune preuve textuelle ${ }^{48}$. Si tel est le cas, on peut alors affirmer que, jusqu'à ce point, sur le plan théorique, il n'y a pas encore eu de «rencontre ». C'est au contraire précisément avec Spinoza et, plus en général, à l'intérieur du courant souterrain du matérialisme que Machiavel «explose » théoriquement dans la pensée d'Althusser. À la lumière du matérialisme aléatoire on pourrait donc relire aussi l'auto-dénonciation par Althusser de son extranéité à la philosophie, avec la revendication d'être "seulement " un agitateur politique en philosophie. C'est un non-philosophe bien étrange que celui-ci, qui produit des effets aussi puissants à l'intérieur et contre la philosophie. L'autodénonciation est presque un calque de l'auto-accusation machiavélienne d'être un homme du peuple qui prétend parler au prince ${ }^{49}$. Autant il faut ne pas être prince pour produire des effets sur le prince, autant il faut ne pas être philosophe pour produire des effets sur la philosophie.

Tout le dispositif du matérialisme aléatoire travaille en vue de cette tâche, pas seulement Machiavel, qui n'est que l'un des atomes qui le composent. Parmi les points saillants de cette attaque matérialiste de la philosophie, il y aura la révolte contre la continuité homogène de l'histoire, c'est-à-dire la production d'une connaissance adéquate du temps historique comme construction de temporalités différentielles: cette idée de « connaissance » comme " construction » passe autant à travers Machiavel qu'à travers Spinoza. Il y aura aussi l'opposition de la contingence non pas à la nécessité, mais à la téléologie. Le vide et les atomes, écrit Althusser dans «Le courant souterrain du matérialisme de la rencontre ", ne fondent pas la liberté, mais sont plutôt la garantie de l'absence d'un plan qui précède leur rencontre. Rien, à l'exception des circonstances factuelles de la rencontre, n'a préparé la rencontre elle-même : ceci est un usage puissant, original et révolutionnaire de Machiavel qui paradoxalement, encore une fois, n'émerge 
pas des écrits consacrés à Machiavel, mais s'affirme avec force lorsque Machiavel est inséré dans le creuset théorique du matérialisme aléatoire.

«Pas plus qu'on ne choisit son temps, on ne choisit ses maitres $»^{50}$. Ainsi écrit Althusser et ainsi l'on doit peut-être interpréter cette rencontre, contingente et nécessaire, entre sa «non-philosophie » et la «non-philosophie » de Machiavel. Affronter cette rencontre est un peu comme affronter celle entre Spinoza et Marx. Il s'agit de rencontres qui donnent un sens au «vide » sur lequel elles s'opèrent, ou mieux qui se produit pendant qu'elles s'opèrent. Le vide est celui d'une " linéarité impossible ». Tout comme la rencontre entre Spinoza et Marx, qui résiste à toute linéarité, à la construction d'une tradition - fût-elle matérialiste -, celle entre Althusser et Machiavel fait aussi partie de ce type de rencontres, qui procèdent souvent par écarts, pertes, mystifications et ambivalences. L'effort et le geste théorique le plus intéressant, justement comme dans le cas de Spinoza et Marx, consistent alors dans le fait de forcer les textes ${ }^{51}$. Dans ce cas, il s'agit de mettre en tension les analyses qu'Althusser fait de Machiavel avec le rôle et le statut que celui-ci revêt à l'intérieur du dispositif global du matérialisme aléatoire. Mettre en lumière cette tension avec les ambivalences du texte althussérien signifie aussi soustraire sa pensée à un usage simplement instrumental et la rendre à sa temporalité articulée. C'est justement cette tension qui, en plus, rend aussi fraîche et actuelle la rencontre entre Machiavel et Althusser. "Je ne suis pas un philosophe - écrivait-il dans la lettre à Franca - et pourtant il faudrait l'être (...). Mais ce n'est pas pour moi. Je sais trop peu de choses, et n'ai plus le temps de les apprendre ». Peut-être cette incomplétude est précisément le chiffre de la rencontre avec Machiavel qui, dans l'avant-propos au premier livre des Discours, écrivait : «Et si le talent insuffisant, le peu d'expérience des choses présentes et la faible connaissance des choses antiques doivent rendre ma tentative imparfaite et de peu d'utilité, ils ouvriront du moins le chemin à quelqu'un qui, avec plus de vertu, de discours et de jugement, pourra satisfaire mon intention $»^{52}$.

\section{NOTES}

1. «Je suis venu à Machiavel par un mot, sans cesse répété, de Marx disant que le capitalisme est né de la rencontre "entre l'homme aux écus et les travailleurs libres", libres c'est-à-dire dépouillés de tout, de leurs moyens de travail, de leur logis et de leur famille, dans la grande expropriation des campagnes anglaises (c'était là son exemple de prédilection). Rencontre » (L. Althusser, «L'unique tradition matérialiste », in Lignes, 18, 1993, p. 91).

2. «Je me mis à lire un peu de Gramsci (sur les intellectuels) mais interrompit vite ma lecture pour m'engager dans celle de Machiavel » (ibid.).

3. L. Althusser, Lettres à Franca (1961-1973), Paris, Stock/IMEC, 1998, le 12 juillet 1967, p. 750. Et encore depuis Gordes, en août 1967 : «Dommage que la philosophie m'intéresse à un âge aussi avancé, si tard dans la vie, et sans rien d'accumulé derrière moi. Je n'ai qu'une sorte de "flair" clinique, et quelques connaissances de détail, ce qui ne suffit pas à faire un philosophe, tout au plus un agitateur philosophique » (ibid., p. 751).

4. L. Althusser, Politique et Histoire. De Machiavel à Marx. Cours à l'École normale supérieure 1955-1972, Paris, Seuil, 2006. 
5. Ibid., p. 199-200.

6. "Je conclurais donc volontiers que l'absence de déduction génétique des formes sociales et politiques à partir d'une théorie de la nature humaine dénonce le caractère factice de l'anthropologie machiavélienne. Disons : il s'est donné en fait d'anthropologie juste ce qu'il lui fallait de contenu et de concept (le désir infini) pour rejeter toute anthropologie éthique ou religieuse; il ne s'est pas donné assez de concepts ni de peine pour fonder sur eux sa théorie politique pour cette raison fondamentale que sous l'apparence superficielle d'une anthropologie (ou d'une théorie de la nature humaine), il décrit en fait des comportements politiques et sociaux. Son anthropologie reste, dans la mesure où elle existe, négative et critique » (ibid., p. 240).

7. «[La pensée de Machiavel] est incapable de se fonder soit dans une anthropologie, soit dans une théorie cyclique de l'histoire (elle-même d'ailleurs de nature anthropologique). Cette double exclusion et de l'objet classique de la philosophie politique, et de l'opération de fondement théorique de ses descriptions et de ses conclusions politiques, fait toute la solitude de Machiavel (... )»(ibid., p. 244).

8. Idem.

9. Sur la conception de la nature humaine chez Machiavel cf. E. Namer, « La nature humaine chez Machiavel », in Les Études Philosophiques, 16, 1961, p.307-312; M. Tlili, "Méchanceté de l'homme ", in Revue de Métaphysique et de Morale, 73, 1968, p. 205-222, p. 205-222 ; M. F. Sciacca, «La concezione dell'uomo », in Cultura e Scuola, 33-34, 1970, pp. 56-71; S. Zeppi, « Il pessimismo antropologico nel Machiavelli del periodo anteriore ai "Discorsi" ", in Filosofia politica, 6, 1992, p. 193-242. Sur le rapport avec Hobbes cf. par exemple Chr. Lazzeri, « Les racines de la volonté de puissance: le "passage" de Machiavel à Hobbes ", in Y. C. Zarka-J. Bernhardt (éds.), Thomas Hobbes. Philosophie première, théorie de la science et politique, Paris, Presses Universitaires de France, 1990, p. 225-246. Sur la comparaison avec Spinoza, en plus de V. Morfino, Il tempo e l'occasione. L'incontro Spinoza-Machiavelli, Milano, Led, 2002 et L. Bove, La stratégie du conatus. Affirmation et résistance chez Spinoza, Paris, Vrin, 1996, je me permets de renvoyer à mon Tumultes et indignation. Conflit, droit et multitude chez Machiavel et Spinoza, Paris, Éd. Amsterdam, 2010.

10. "Nous connaissons tous la Section VIII du Livre I du Capital, où Marx s'attaque à la prétendue "accumulation originelle" (traduite: "primitive"). Dans cette accumulation originelle les idéologues du capitalisme racontaient l'histoire édifiante du capital, comme les philosophes du droit naturel racontent l'histoire de l'État. Au début il y avait un travailleur indépendant, qui avait tant d'ardeur au travail et d'esprit d'économie qu'il put épargner puis échanger. Comme un pauvre passait, il lui rendit le service de le nourrir en échange de son travail, générosité qui lui permit d'accroître son acquis et de rendre par son bien accru d'autres services du même genre à d'autres malheureux. D'où l'accumulation du capital : par le travail, l'ascèse et la générosité. Nous savons comment Marx répond: par l'histoire des pillages, des vols, des exactions, par la dépossession violente des paysans anglais chassés de leurs terres, et leurs fermes détruites pour qu'ils soient à la rue, par une tout autre histoire autrement saisissante que la rengaine moralisante des idéologues du capitalisme » (L. Althusser, «Solitude de Machiavel » in Solitude de Machiavel et autres textes, Paris, Presses Universitaires de France, 1998, p. 319).

11. Ibid., p. 320.

12. Cf. par exemple B. Spinoza, Traité politique, traduit par E. Saisset, revu par L. Bove, Paris, Le Livre de Poche, 2002, I, 1.

13. «Il arrive plutôt que, dans ces vicissitudes, manquant toujours de sagesse et de forces, un État soit assujetti par un État voisin, mieux ordonné que lui ; mais en admettant que cela n'arrive pas, un État pourrait tourner à l'infini dans ces gouvernements" (N. Machiavel, Discours sur la première décade de Tite-Live, traduit par A. Fontana et X. Tabet, Paris, Gallimard, 2004, I, 2, p. 63).

14. "Considérant donc tout cela, on voit comment il était nécessaire aux législateurs de Rome, s'ils voulaient que Rome restât tranquille que ces deux républiques, de faire l'une de ces deux choses : soit ne pas employer la plèbe à la guerre, comme les Vénitiens, soit ne pas ouvrir la voie 
aux étrangers, comme les Spartiates. Et les Romains firent l'une et l'autre chose, ce qui permit à la plèbe de se renforcer et de s'accroître, et lui fournit d'innombrables occasions de provoquer des tumultes. Mais, si l'état romain devenait plus tranquille, l'inconvénient qui en résultait était qu'il devenait aussi plus faible, car il se fermait alors la voie qui pouvait le mener à la grandeur qu'il atteignit; de sorte que si Rome voulait éliminer les causes de tumultes, elle éliminait aussi celles de son agrandissement » (ibid., I, 6, p. 79-80).

15. Dans les Histoires florentines, Machiavel écrit encore : « Le but de tous ceux qui entreprennent une guerre a toujours été et, raisonnablement, doit être de s'enrichir soi-même et d'appauvrir son ennemi ; on ne doit chercher dans une victoire ou une conquête qu'à accroître sa puissance et affaiblir celle de l'adversaire » (N. Machiavel, Histoires florentines, in Euvres complètes, texte présenté et annoté par E. Barincou, Paris, Gallimard, 1952, VI, 1, p. 1228). C'est un principe qui vaut aussi bien sur le plan extérieur, que sur celui intérieur, dans le conflit entre nobles et peuple. Althusser néglige significativement les Histoires florentines par rapport aux Discours et au Prince.

16. L. Althusser, Politique et Histoire, op. cit., p. 243.

17. Voir en particulier le livre VI des Histoires de Polybe et le livre IV de la Politique d'Aristote.

18. L. Althusser, « Machiavel et nous ", in Écrits philosophiques et politiques, t. II, Paris, Stock/Imec, 1995, p. 83.

19. Ibid., p. 59-60.

20. «Mais à considérer Cyrus et les autres qui ont acquis ou fondé des royaumes, vous constaterez qu'ils sont tous admirables; et si l'on considère leurs actions et leurs ordres particuliers, ils ne paraitront pas discordants par rapport à ceux de Moïse, qui eut un si grand précepteur. Et, si on examine leurs actions et leur vie, on ne voit pas qu'ils aient reçu rien d'autre de la fortune que l'occasion, qui leur donna la matière pour pouvoir y introduire cette forme qui leur parut bonne : et, sans cette occasion, la vertu de leur esprit se serait éteinte et, sans cette vertu, l'occasion serait venue en vain » (N. Machiavel, Le Prince, trad. par J.-L. Fournel et J.-Cl. Zancarini, Paris, Presses Universitaires de France (coll. Quadrige), 2014, VI, p. 113).

21. «Une fois donc considérées toutes les choses examinées ci-dessus, et me demandant, en moimême, si présentement en Italie couraient des temps capables d'honorer un nouveau prince et s'il y avait là matière à donner occasion à un homme prudent et vertueux d'y introduire une forme qui lui fît honneur à lui-même et du bien à la communauté des hommes, il me semble que tant de choses y concourent, au bénéfice d'un prince nouveau, que, moi, je ne sais pas s'il y eût jamais un temps plus apte à cela » (ibid., XXVI, p. 265-267).

22. L. Althusser, Politique et Histoire, op. cit., p. 208.

23. L. Althusser, Machiavel et nous, op. cit., p. 61.

24. Ibid., p. 54.

25. Ibid.

26. Cf. W. Montag, "Beyond Force and Consent: Althusser, Spinoza, Hobbes ", in A. Callari-D. F. Ruccio (éd.), Postmodern Materialism and the Future of Marxist Theory, Hanover and London, Wesleyan University Press, 1996, p. 91-106.

27. L. Althusser, « Machiavel et nous », op. cit., p. 97.

28. Ibid., p. 86-87.

29. « On comprend que le programme ou la tendance consistant à unir la ville et la campagne n'ait pu avoir chez Machiavel qu'une expression militaire, si l'on réfléchit au fait que le jacobinisme français serait inexplicable sans le présupposé de la culture des physiocrates, avec sa démonstration de l'importance économique et sociale du cultivateur direct. Les théories économiques de Machiavel ont été étudiées par Gino Arias (dans les Annali d'Economia de l'université Bocconi), mais il faut se demander si Machiavel a eu des théories économiques : il faudra voir si le langage essentiellement politique de Machiavel peut être traduit en termes économiques et à quel système économique on peut le réduire. Voir si Machiavel, qui vivait à 
l'époque du mercantilisme a précédé son temps sur le plan politique et s'il a anticipé sur certaines exigences qui ont trouvé plus tard leur expression chez les physiocrates » (A. Gramsci, Cahiers de prison, Cahiers 10,11, 12 et 13, traduit par P. Fulchignoni, G. Granel et N. Negri, Avantpropos, notice et notes de R. Paris, Paris, Gallimard, 1978, p. 372-373).

30. «Gramsci, dunque, riprende Croce e si contrappone a lui. Lo riprende in quanto ha fatto compiere un progresso decisivo alla machiavellistica, sgomberando il campo da tutta una serie di "problemi falsi e inesistenti", come quello della presunta "immoralità" di Machiavelli. Ma vi si contrappone in quanto ha poi costretto la portata filosofica della politica-pratica nell'ambito del sistema delle categorie, limitando, in tal modo, anche il valore filosofico di Marx da lui non a caso definito "Machiavelli del proletariato". Si può dire pertanto che, in un certo senso, Gramsci rovescia la prospettiva crociana, in quanto scorge proprio nella politica la scoperta in nuce di un'intera nuova filosofia, rispetto alla quale Marx, cioè le Tesi su Feuerbach, rappresentano uno sviluppo conseguente e non una rottura : "La innovazione fondamentale introdotta da Marx nella scienza politica e storica in confronto del Machiavelli è la dimostrazione che non esiste una 'natura umana' fissa e immutabile" ( F. Frosini, Gramsci e la filosofia. Saggio sui "Quaderni del carcere ", Roma, Carocci, 2003, p. 164-165).

31. A. Gramsci, Cahiers de prison, op. cit., p. 385-386.

32. L'exemple le plus significatif et célèbre de cette "préparation» est, naturellement, la métaphore du fleuve et des barrages dans Le Prince, XXV : «Pour que notre libre arbitre ne soit pas éteint, j'estime qu'il peut être vrai que la fortune soit l'arbitre de la moitié de nos actions, mais que etiam elle nous en laisse gouverner l'autre moitié, ou à peu près. Et je la compare à l'un de ces fleuves furieux qui, lorsqu'ils se mettent en colère, inondent les plaines, ruinent édifices et arbres, arrachent la terre, d'un côté, et la déposent, de l'autre : chacun s'enfuit devant eux, chacun cède à leur assaut, sans pouvoir, en aucun point, y faire obstacle. Et bien qu'ils soient ainsi faits, il n'en reste pas moins que les hommes, quand les temps sont calmes, pourraient y pourvoir par des remparts et des digues; de façon que, quand viendrait ensuite la crue, ou bien ils s'en iraient par quelque canal, ou bien leur assaut ne serait ni aussi dommageable ni aussi effréné. Il en va semblablement de la fortune, qui montre sa puissance là où il n'est pas de vertu ordonnée pour lui résister : et elle tourne ses assauts là où elle sait qu'on n'a fait ni digue ni rempart pour la contenir» (N. Machiavel, Le Prince, op. cit., XXV, p. 259-261).

33. «Dans son précis d'histoire de la Révolution française (éd. Colin), Mathiez, s'opposant à l'histoire traditionnelle vulgaire qui "trouve" a priori chaque fois une crise qui coïncide avec les grandes ruptures de l'équilibre social, affirme que vers 1789 la situation économique immédiate était plutôt bonne, en sorte qu'on ne peut dire que l'effondrement de l'État absolu soit dû à une crise d'appauvrissement » (A. Gramsci, Cahiers de prison, op. cit., p. 384).

34. Sur le concept d'immanence chez Gramsci cf. F. Frosini, «Immanenza e materialismo storico nei "Quaderni del carcere" di Gramsci », in Quaderni materialisti, 5, 2006, pp. 147-158.

35. L'expression italienne est " principio di parte» et signifie que le prince est une partie et non pas le tout de la politique, ce qui permet ainsi d'insister sur la nature conflictuelle de la politique $(\mathrm{NdT})$.

36. A. Gramsci, Cahiers de prison, op. cit., p. 355-356.

37. Cette interprétation et, plus en général, le rapport entre Le Prince et les Discours, est encore au centre du débat historiographique, où l'on tend parfois à faire émerger les aspects les plus « crus » du réalisme machiavélien et d'autres fois à le neutraliser, en le reléguant - bien que dans une position de protagoniste - à l'intérieur du filon de l'humanisme tardif et du républicanisme civil. Cf. M. Viroli, Machiavelli, Oxford University Press, Oxford, 1998.

38. Je me permets de renvoyer encore une fois à mon F. Del Lucchese, Tumultes et indignation, op. cit. Cf. contra M. Viroli, Machiavelli, op. cit. ou G. Sfez, Machiavel, le prince sans qualités, Paris, Kimé, 1998. 
39. "Machiavelli addresses the masses via the silhouette of a resolute ruler only because, conceived in that form, decisive action can begin at any time. The Prince, unlike the proletariat, is "the pure possibility of the event", "agency out of the void", "absolute new beginning". Machiavelli's writings analyse "the conditions of an impossible event", blasting open the continuum of history. It is as if we overhear at this point an uncanny, voluntarist echo of Benjamin» (G. Balakrishnan, "From Florence to Moscow», in New Left Review, 2000, 3, p. 158-164).

40. L. Althusser, Machiavel et nous, op. cit., p. 70.

41. «Il reste pourtant ce fait, irrécusable, que Machiavel n'a pas écrit un texte comparable au Manifeste communiste » (ibid., p. 69).

42. E. Terray, « Une rencontre : Althusser et Machiavel », in S. Lazarus (éd.), Politique et philosophie dans l'œuvre de Louis Althusser, Paris, Presses Universitaires de France, 1993.

43. Une lecture de ce genre, basée sur l'idée du dépassement et de la limitation nécessaires de la violence naturelle par des bornes institutionnelles, avait déjà été avancée par N. Badaloni, « Natura et società in Machiavelli », in Studi Storici, 10, 1969, pp. 675-708.

44. Cf. M.E. Vatter, « Althusser et Machiavel: la politique après la critique de Marx », in Multitudes , 13, 2003, pp. 151-163; «Legality and Resistance: Arendt and Negri on Constituent Power ", in Kairos, 20, 2002, pp. 191-230; «Machiavelli After Marx: the Self-Overcoming of Marxism in the Late Althusser ", Theory and Event, 7, 2004. Le texte le plus important de Vatter est Between Form and Event: Machiavelli's Theory of Political Freedom, Dordrecht-Boston-London, Kluwer Academic Publisher, 2000.

45. Cf. N. Machiavel, Discours, op. cit., I, 5.

46. $\mathrm{Ou}$ du pouvoir constituant chez Antonio Negri, que Vatter semble considérer comme entièrement superposable à la puissance chez Spinoza. Cf. A. Negri, Le pouvoir constituant. Essai sur les alternatives de la modernité, Paris, Presses Universitaires de France, 1997.

47. M. E. Vatter, « Legality and Resistance », op. cit., p. 221.

48. E. Terray, «Une rencontre : Althusser et Machiavel », op. cit., p. 141.

49. «Et je ne veux pas que l'on impute à la présomption qu'un homme de bas et infime état ait la hardiesse d'examiner les gouvernements des princes et de leur donner des règles; en effet, de même que ceux qui dessinent les pays se placent en bas, dans la plaine, pour considérer la nature des monts et des lieux élevés, et que, pour considérer celle des lieux d'en bas, ils se placent haut sur les monts, semblablement, pour connaître bien la nature des peuples, il faut être prince, et pour connaître bien celle des princes, il convient d'être du peuple » (N. Machiavel, Le Prince, op. cit., «Proemio », p. 77).

50. L. Althusser, L'avenir dure longtemps suivi de Les faits, Paris, Stock/IMEC, 1992, p. 326.

51. Cf. dans ce sens, le dispositif théorique global de V. Morfino, Il tempo della moltitudine. Materialismo e politica prima e dopo Spinoza, Rome, Manifestolibri, 2005.

52. N. Machiavel, Discours, op. cit., I, p. 49-50.

\section{RÉSUMÉS}

Dans cet article je reconstruis la «rencontre» d'Althusser avec la pensée de Machiavel. Une rencontre qui se fait au nom d'un défi au concept de philosophie et à la nature de la pensée philosophico-politique, à travers les catégories de vertu, conflit, intervention dans la 
conjoncture. Althusser se présente comme un disciple et divulgateur de la pensée de Machiavel. Je vise à montrer, toutefois, que son analyse, loin d'être originale et révolutionnaire dans les premiers écrits, s'approfondit et devient explosive seulement dans les derniers écrits sur le matérialisme de la rencontre.

In questo articolo ricostruisco l'incontro' di Althusser con il pensiero di Machiavelli. Un incontro che si fa nel nome di una sfida al concetto di filosofia e alla natura del pensiero filosofico-politico, atraverso le categorie di virtù, conflitto, intervento nella congiuntura. Althusser si reclama un seguace e un divulgatore del pensiero di Machiavelli. Intendo mostrare, tuttavia, che la sua analisi di Machiavelli, tutt'altro che originale e rivoluzionaria nei primi scritti, si approfondisce e diventa dirompente solo negli ultimi scritti, quelli sul materialismo dell'incontro.

\section{INDEX}

Thèmes : philosophie politique, philosophie française contemporaine, matérialisme, marxisme

Index géographique : Italie, France

Index chronologique : XVI siecle, XX siecle

Mots-clés : Machiavel, Althusser, vertu, fortune, materialisme aleatoire, conflit, marxisme

\section{AUTEURS}

\section{FILIPPO DEL LUCCHESE}

Filippo Del Lucchese est professeur en histoire de la pensée politique à la Brunel University (Londres), chercheur à l'Université de Johannesburg et directeur de programme au Collège International de Philosophie à Paris. Il est membre du comité éditorial de Décalages (Los Angeles). 